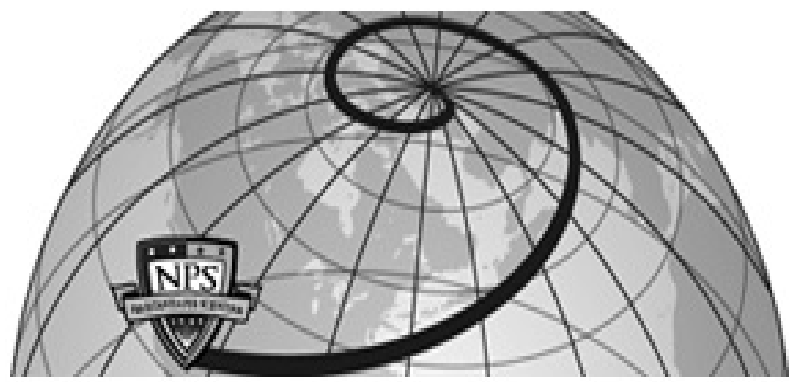

Calhoun: The NPS Institutional Archive DSpace Repository

\title{
NPSAT1 Parameter Estimation Using Unscented Kalman Filtering
}

Sekhavat, Pooya; Ross, I. Michael; Gong, Qi IEEE

https://hdl.handle.net/10945/29662

This publication is a work of the U.S. Government as defined in Title 17, United States Code, Section 101. Copyright protection is not available for this work in the United States.

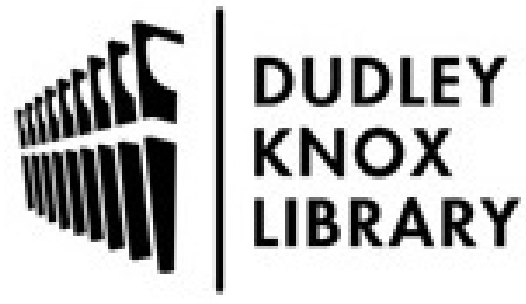

http://www.nps.edu/library
Calhoun is the Naval Postgraduate School's public access digital repository for research materials and institutional publications created by the NPS community. Calhoun is named for Professor of Mathematics Guy K. Calhoun, NPS's first appointed -- and published -- scholarly author.

Dudley Knox Library / Naval Postgraduate School 411 Dyer Road / 1 University Circle Monterey, California USA 93943 


\title{
NPSAT1 Parameter Estimation Using Unscented Kalman Filtering
}

\author{
Pooya Sekhavat, Qi Gong and I. Michael Ross
}

\begin{abstract}
NPSAT1 is a small satellite being built at the Naval Postgraduate School and scheduled to launch in 2007. It primarily employs magnetic sensing and actuation for attitude control. The nature of the in-house fabrication and assembly of the spacecraft requires reliable computational estimation of the difficult-to-measure parameters of the end-product. The inherent nonlinear dynamics of the system makes the observer design a challenging problem. This paper presents the successful implementation of the Unscented Kalman Filter (UKF) for the spacecraft parameter estimation. Since a three-axis magnetometer is the only sensor onboard, the UKF algorithm also estimates the system orientation and angular velocity. The unit quaternion constraint is enforced by treating the norm of the quaternions as a dummy measurement. Simulations and ground test experimental results show the superior performance of the UKF in spacecraft dual state-parameter estimation.
\end{abstract}

\section{INTRODUCTION}

NPSAT1 is a small prolate non-spinning satellite that primarily uses a three-axis active magnetic attitude control. It is designed and constructed at the Naval Postgraduate School and is scheduled to launch in 2007 at an altitude of $600 \pm 40$ km (Fig. 1). Magnetic attitude control provides robustness, light weight, lower power consumption, and cost-efficiency and is an attractive choice for low-orbit satellites. The control system includes a magnetometer and three magnetic torque rods. Interaction between the three magnetic dipole moments generated by the torque rods and the Earth magnetic field produces a resulting torque that actuates the spacecraft.

In order to be able to design and verify the effectiveness of the spacecraft attitude control system, a hardware-in-theloop air-bearing platform with all actual NPSAT1 sensors and actuators was designed to mimic NPSAT1 attitude movement in a laboratory environment. Similar to the actual NPSAT1, the dynamic model of the ground test-bed includes mass properties and moments of inertia. However, unlike the inflight motion, the airbearing table moves about a center of rotation located slightly above the center of mass to provide passive stability. This introduces an additional parameter (distance between center of mass and center of rotation) into the dynamic model that should be determined for attitude control design.

By designing the spacecraft to be symmetrical and aligning the coordinate system with the spacecraft principal axes, the products of inertia vanish and the number of remaining moments of inertia that should be measured/estimated reduces to

Department of Mech. \& Astro. Eng., Naval Postgraduate School, Monterey, CA 93943, E-mail: psekhava@nps.edu.

Department of Elec. \& Comp. Eng., Univ. of Texas at San Antonio, 78249, E-mail: qi.gong@utsa.edu.

Department of Mech. \& Astro. Eng., Naval Postgraduate School, Monterey, CA, 93943, Email: imross@nps.edu

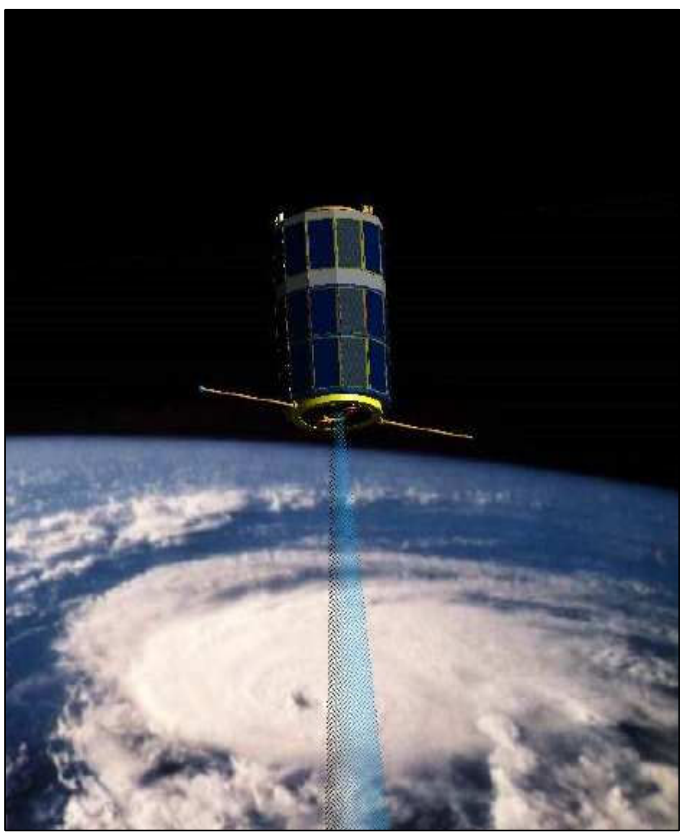

Fig. 1. Artist's Rendition of NPSAT1

the three principal moments of inertia. One way of finding the values of the moments of inertia is to mathematically calculate the overall system's moments of inertia knowing the inertia values (geometry) and exact locations of each system component. Although this approach can be of some help for simulation-based studies, it has little or no practicality when it comes to real situations. In practice, the system parameters can alternatively be estimated using various parameter identification techniques that employ sensor measurements and known physical equations. For the system under study, an example of such practice would be to measure the swing period of the airbearing table under some known (or zero) control. Finding the distance between center of mass and center of rotation using such strictly experimental approaches would, for example, include measurement of inclination angle when the system is counter-balanced at an inclined position with a known static weight placed at a known distance from the center of rotation.

A third fundamentally different approach to estimate the system parameters is to use filtering techniques with capability of simultaneous state and parameter estimation. One of the most powerful estimation techniques that has been commonly used in various applications is the Kalman filter and its later extensions to account for system nonlinearities [1], [2], [3]. Through linearizing the system dynamics along 
the actual (nonlinear) trajectory and applying the standard Kalman Filter, the Extended Kalman Filter (EKF) can capture the nonlinear characteristics of the system during the estimation. However, the inherent linearization in the scheme can be the cause of divergence and ultimate failure of the scheme. Also, the method's Jacobian matrix derivation can be cumbersome and prone to human errors.

The more recent alternative estimation method proposed by Julier and Uhlmann is the Unscented Kalman filter (UKF) [4], [5]. It is "founded on the intuition that it is easier to approximate a probability distribution than it is to approximate an arbitrary nonlinear function or transformation" [4]. By introducing a set of so-called sigma-points to capture the higher order statistics of the system, UKF successfully avoids the linearization step. This property alone is a significant advantage over the EKF and accommodates nonlinear systems with discontinuity, table-looking parameters, and even rule-based decision making problems, where EKF is not applicable. By preserving the higher order information of the system, UKF improves both accuracy and convergence properties of the solution. Remarkably, UKF has roughly the same computational complexity as the EKF [6], [7] and its superior performance carries no additional computational burden.

Following successful UKF applications for spacecraft attitude estimation [6], [8], [9], the method was successfully employed for NPSAT1 state estimation [10]. This paper extends the previous work by examining the UKF method for simultaneous parameter-state estimation.

Kalman filtering methods for state-parameter estimation can be grouped into two major categories: joint and dual. They both estimate the state and parameters concurrently. In the joint estimation approach, unknown parameters are augmented to the state vector [11], [12] and the time-update of the augmented part of the state vector allows for no changes beyond the effects of process noise. That means parameter values are updated throughout the process of propagating the entire augmented covariance matrix. Dual filtering technique intertwines a pair of distinct sequential filters, one estimating the states and the other estimating the parameters [8], [13], [14]. The method is capable of decoupling the parameter filter from the state filter. This can provide more designer flexibility to choose the covariance matrices in a way that reduces the detrimental effects of the state filter on the parameter filter and vice versa. In this work, we employ the dual state-parameter estimation technique with the goal of estimating the unknown difficultto-measure parameters of NPSAT1 airbearing platform.

The paper is organized as follows. First, NPSAT1 attitude dynamics is explained followed by a brief review of the Unscented Kalman Filter and its application to dual stateparameter estimation. Next, UKF is implemented for the airbearing parameter estimation and the simulation results are presented. Finally, the method is examined using the actual sensor measurements from NPSAT1 ground tests on the airbearing platform. To ensure the state convergence, the estimated states are compared with an alternative set of independently measured position values from an optical position measurement system installed on the test rig. The convergence of parameter estimation is validated through starting the estimation routine from various initial conditions. It is shown that simulations and experimental ground test results are in excellent agreement and UKF can be successfully used to estimate the states and parameters of such a highly nonlinear dynamic system.

\section{Dynamic Model of the System}

\section{A. NPSAT1 Dynamic Model}

In this section we briefly describe the dynamics of the NPSAT1 spacecraft. More details can be found in Refs [10], [15]. By defining the standard quaternions and body rates as the state variables, we have $x=(q, \omega) \in \mathbb{R}^{7}$, where

- $q=\left(q_{1}, q_{2}, q_{3}, q_{4}\right):$ quaternion of the body frame with respect to the orbit frame,

- $\omega=\left(\omega_{x}, \omega_{y}, \omega_{z}\right)$ : rotation rate of the body frame with respect to the inertial frame expressed in the body frame.

The quaternion vector must lie on $S^{3}$ given by the following state constraint:

$$
q_{1}^{2}+q_{2}^{2}+q_{3}^{2}+q_{4}^{2}=1
$$

The kinematic equations of motion for the NPSAT1 can now be expressed as [15]:

$$
\begin{aligned}
& \dot{q}_{1}=\frac{1}{2}\left[\omega_{x} q_{4}-\omega_{y} q_{3}+\omega_{z} q_{2}+\omega_{0} q_{3}\right] \\
& \dot{q}_{2}=\frac{1}{2}\left[\omega_{x} q_{3}+\omega_{y} q_{4}-\omega_{z} q_{1}+\omega_{0} q_{4}\right] \\
& \dot{q}_{3}=\frac{1}{2}\left[-\omega_{x} q_{2}+\omega_{y} q_{1}+\omega_{z} q_{4}-\omega_{0} q_{1}\right] \\
& \dot{q}_{4}=\frac{1}{2}\left[-\omega_{x} q_{1}-\omega_{y} q_{2}-\omega_{z} q_{3}-\omega_{0} q_{2}\right]
\end{aligned}
$$

where $\omega_{0}$ is the angular velocity of the orbit with respect to the inertial frame.

Control is applied through the interaction of the dipole moments generated by the three magnetic torque rods $u=$ $\left(u_{1}, u_{2}, u_{3}\right) \in \mathbb{R}^{3}$ and the Earth magnetic field. Choosing $\left(I_{1}, I_{2}, I_{3}\right)$ as the principal moments of inertia, $\mu$ as Earth gravitational constant, and $r_{0}$ as the distance from the center of spacecraft to the center of the Earth, the Euler's dynamic equations of motion are:

$$
\begin{aligned}
\dot{\omega}_{x}= & \frac{I_{2}-I_{3}}{I_{1}}\left[\omega_{y} \omega_{z}-3 \frac{\mu}{r_{0}^{3}} C_{23} C_{33}\right]+ \\
& \frac{1}{I_{1}}\left[B_{z}(q, t) u_{2}-B_{y}(q, t) u_{3}\right] \\
\dot{\omega}_{y}= & \frac{I_{3}-I_{1}}{I_{2}}\left[\omega_{x} \omega_{z}-3 \frac{\mu}{r_{0}^{3}} C_{13} C_{33}\right]+ \\
& \frac{1}{I_{2}}\left[B_{x}(q, t) u_{3}-B_{z}(q, t) u_{1}\right] \\
\dot{\omega}_{z}= & \frac{I_{1}-I_{2}}{I_{3}}\left[\omega_{x} \omega_{y}-3 \frac{\mu}{r_{0}^{3}} C_{13} C_{23}\right]+ \\
& \frac{1}{I_{3}}\left[B_{y}(q, t) u_{1}-B_{x}(q, t) u_{2}\right]
\end{aligned}
$$


where $C_{i j}$ denote the corresponding element in the Direction Cosine Matrix, $C_{q}$, defined as:

$$
\left[\begin{array}{c}
q_{1}^{2}-q_{2}^{2}-q_{3}^{2}+q_{4}^{2}, 2\left(q_{1} q_{2}+q_{3} q_{4}\right), 2\left(q_{1} q_{3}-q_{2} q_{4}\right) \\
2\left(q_{1} q_{2}-q_{3} q_{4}\right), q_{2}^{2}-q_{1}^{2}-q_{3}^{2}+q_{4}^{2}, 2\left(q_{2} q_{3}+q_{1} q_{4}\right) \\
2\left(q_{1} q_{3}+q_{2} q_{4}\right), 2\left(q_{2} q_{3}-q_{1} q_{4}\right), q_{3}^{2}-q_{1}^{2}-q_{2}^{2}+q_{4}^{2}
\end{array}\right] .
$$

The vector $\left(B_{x}, B_{y}, B_{z}\right)$ is the Earth magnetic field in the body frame and can be defined as

$$
\left(\begin{array}{c}
B_{x}(q, t) \\
B_{y}(q, t) \\
B_{z}(q, t)
\end{array}\right)=C_{q}\left(\begin{array}{c}
B_{1}(t) \\
B_{2}(t) \\
B_{3}(t)
\end{array}\right)
$$

where $\left(B_{1}(t), B_{2}(t), B_{3}(t)\right.$ are the components of the Earth magnetic field in the orbit frame [16]:

$$
\begin{aligned}
B_{1}= & \frac{M_{e}}{r_{0}^{3}}\left[\cos \left(\omega_{0} t\right)\left[\cos (\epsilon) \sin (i)-\sin (\epsilon) \cos (i) \cos \left(\omega_{e} t\right)\right]\right. \\
& \left.-\sin \left(\omega_{0} t\right) \sin (\epsilon) \sin \left(\omega_{e} t\right)\right] \\
B_{2}= & -\frac{M_{e}}{r_{0}^{3}}\left[\cos (\epsilon) \cos (i)+\sin (\epsilon) \sin (i) \cos \left(\omega_{e} t\right)\right] \\
B_{3}= & \frac{2 M_{e}}{r_{0}^{3}}\left[\sin \left(\omega_{0} t\right)\left[\cos (\epsilon) \sin (i)-\sin (\epsilon) \cos (i) \cos \left(\omega_{e} t\right)\right]\right. \\
& \left.+2 \cos \left(\omega_{0} t\right) \sin (\epsilon) \sin \left(\omega_{e} t\right)\right] .
\end{aligned}
$$

For NPSAT1, the known parameters are:

- $\omega_{0}=\sqrt{\frac{\mu}{r_{0}^{3}}} \approx 0.00108 \mathrm{rad} / \mathrm{s}$;

- $\mu=3.98601 \times 10^{14} \mathrm{~m}^{3} / \mathrm{s}^{2}$; the Earth gravitational constant.

- $M_{e}=7.943 \times 10^{15}$ Wb.m; the magnetic dipole moment of the Earth.

- $r_{0}=6978$; the distance from the center of spacecraft to the center of the Earth (a constant for a circular orbit).

- $i=35.4^{\circ}$; the orbit inclination

- $\epsilon=11.7^{\circ}$; the magnetic dipole tilt

- $\omega_{e}=7.29 \times 10^{-5} \mathrm{rad} / \mathrm{s}$; the spin rate of the Earth.

The complete dynamic model of NPSAT 1 is formulated by equations (2)-(8). Clearly, it is a fairly complex nonlinear system. Note also that as a result of the changes of the magnetic field in time, $\left(B_{1}(t), B_{2}(t), B_{3}(t)\right)$, the overall system is time-variant.

None of the state variables, $(q, \omega)$, can be directly measured as the only onboard sensor is a three-axis magnetometer that measures the magnetic field in the body frame. Since attitude control is done through magnetic actuation, the magnetic field generated by torque rods can also be picked up by the magnetometer. Therefore, the real sensor measurement is the combination of Earth magnetic field, $\left(B_{x}, B_{y}, B_{z}\right)$, and the magnetic field generated by the torque rods. Mathematically speaking, the output function of the system (2)-(8) is

$$
h(q, \omega, u, t)=\left(\begin{array}{c}
B_{x}(q, t) \\
B_{y}(q, t) \\
B_{z}(q, t)
\end{array}\right)+\left(\begin{array}{c}
B_{x u}\left(u_{1}, u_{2}, u_{3}\right) \\
B_{y u}\left(u_{1}, u_{2}, u_{3}\right) \\
B_{z u}\left(u_{1}, u_{2}, u_{3}\right)
\end{array}\right)
$$

where $\left(B_{x u}, B_{y u}, B_{z u}\right)$ denote the magnetic field generated by the torque rods as functions of $\left(u_{1}, u_{2}, u_{3}\right)$. The slopes of these linear functions depend on the relative position of the magnetometer and the torque rods, and can be either directly calculated or determined experimentally. For NPSAT1 airbearing platform, we have experimentally determined that,

$$
\begin{aligned}
& B_{x u}\left(u_{1}, u_{2}, u_{3}\right)=-0.0012 u_{1}-0.000039 u_{2}-0.00051 u_{3} \\
& B_{y u}\left(u_{1}, u_{2}, u_{3}\right)=-0.000013 u_{1}-0.0017 u_{2}-0.00011 u_{3} \\
& B_{z u}\left(u_{1}, u_{2}, u_{3}\right)=0.000049 u_{1}-0.00022 u_{2}+0.0017 u_{3}
\end{aligned}
$$

\section{B. Airbearing-Specific Dynamic Modifications}

As a result of using the air-bearing platform for NPSAT1 ground tests, the equations of motion should represent the system dynamic behavior on Earth. The main difference between the two in-flight and on-Earth motions is the fact that the NPSAT1 center of mass in flight coincides with its center of rotation whereas the center of rotation of the airbearing table on Earth is slightly above its center of mass. This offset, $l$, causes an additional gravity torque modeled as:

$$
\left[T_{x}, T_{y}, T_{z}\right]=\operatorname{mgl}\left[-C_{23}, C_{13}, 0\right],
$$

where $\mathrm{m}$ is the mass of the table platform, $g$ is the gravitational constant on Earth and $l$ is the distance between the table's center of mass and center of rotation. Consequently, in order to capture the true dynamics of the ground test-bed, the dynamic equations (6)-(8) is modified to:

$$
\begin{aligned}
\dot{\omega}_{x}= & \frac{1}{I_{1}}\left[\left(I_{2}-I_{3}\right) \omega_{y} \omega_{z}-m g l C_{23}+\right. \\
& \left.B_{z}(q, t) u_{2}-B_{y}(q, t) u_{3}\right] \\
\dot{\omega}_{y}= & \frac{1}{I_{2}}\left[\left(I_{3}-I_{1}\right) \omega_{x} \omega_{z}+m g l C_{13}+\right. \\
& \left.B_{x}(q, t) u_{3}-B_{z}(q, t) u_{1}\right] \\
\dot{\omega}_{z}= & \frac{1}{I_{3}}\left[\left(I_{1}-I_{2}\right) \omega_{x} \omega_{y}+\right. \\
& \left.B_{y}(q, t) u_{1}-B_{x}(q, t) u_{2}\right]
\end{aligned}
$$

Equations (11) - (13) together with (2) - (5) constitute the complete dynamic model for the airbearing test-bed. Therefore, the parameter estimation scheme should not only estimate the system's moments of inertia, but also the additional constant $m g l$ that is specific to the ground test experiments.

\section{Dual State-Parameter Estimation Using UnSCENTED KaLMAN FILTER}

This section presents a brief overview of the UKF-based dual state-parameter estimation of the NPSAT1 spacecraft. UKF utilizes the so-called sigma points to generate state and parameter predictions at each step. Sigma points are propagated using the system's nonlinear dynamics and the posterior mean and covariance are then calculated from the propagated sigma points. This, preserves the system's both first- and second-order statistics and, compared to EKF, results in a considerably improved convergence properties. In a dual state-parameter estimation routine, the state and parameter estimators are sequentially intertwined in a way that each uses the information generated by the other one for its next update. 


\section{A. State Estimation}

The standard UKF estimation routine is well documented elsewhere [4], [5], [6] and will not be repeated for the sake of brevity. However, UKF in its standard format cannot be implemented for quaternion-based attitude estimation. This is due to the fact that the algebraic unit quaternion constraint [see eq (1)] should be enforced throughout the estimation to prevent the covariance matrix of the quaternion states from becoming singular [1]. As it is detailed in [10], this problem can be remedied by treating the norm of the quaternions as a dummy output and augmenting it to the real measurement vector:

$$
h(q, \omega, u, t)=\left(\begin{array}{c}
B_{x}(q, t)+B_{x u}\left(u_{1}, u_{2}, u_{3}\right) \\
B_{y}(q, t)+B_{y u}\left(u_{1}, u_{2}, u_{3}\right) \\
B_{z}(q, t)+B_{z u}\left(u_{1}, u_{2}, u_{3}\right) \\
q_{1}^{2}+q_{2}^{2}+q_{3}^{2}+q_{4}^{2}-1
\end{array}\right) .
$$

Although there is no real sensor to measure the norm of the quaternions, by definition, we know that the quaternion norm is always one. This simple idea is easy to implement and enforces the algebraic unit constraint at each step. More details on NPSAT1 state estimation using UKF is presented in [10].

\section{B. Parameter Estimation}

The ground test-bed parameters that need to be estimated are $\left[\begin{array}{llll}I_{1} & I_{2} & I_{3} & \mathrm{mgl}\end{array}\right]$ where $I^{\prime} s$ are the second moments of inertia and $m g l$ is the additional constant specific to the airbearing table. The parameter estimation routine is essentially similar to the state estimation and exploits the parameters mean and covariance. The catch is that the predicted output vector for the parameter estimator is in the form of a state vector that is obtained by feeding the parameter sigma points into the previous-step state propagation. The propagated results are then used in conjunction with the current state estimates (that serves as the current measurements) to generate the updated parameter estimates. The updated estimate of the parameters is then fed back into the state estimator for the next iteration. For more details on the method, the readers are referred to Ref. [8], [13], [14].

\section{Simulation Results}

This section presents the simulation results of applying the dual UKF routine for the airbearing parameter estimation. The output (magnetometer measurement) vector is constructed by propagating the system's dynamic equations with parameters $\left(I_{1}, I_{2}, I_{3}, m g l\right)=(2.0,1.5,2.5,0.23)$ from the initial orientation and angular velocity of $\left(q_{1}, q_{2}, q_{3}, q_{4}\right)=(-0.0066,0.0025,-0.467,0.884)$ and $\left(\omega_{x}, \omega_{y}, \omega_{z}\right)=(0,0,0) \mathrm{rad} / \mathrm{s}$, and under a constant control input of $u_{1}=u_{2}=-u_{3}=-33 \mathrm{~A} / \mathrm{m}^{2}$. The UKF estimation starts with the initial orientation, angular velocity, and parameters of $\left(q_{1}, q_{2}, q_{3}, q_{4}\right)=$ $(0,0,0,1),\left(\omega_{x}, \omega_{y}, \omega_{z}\right)=(-0.02,0.02,0.01) \mathrm{rad} / \mathrm{s}$, and $\left(I_{1}, I_{2}, I_{3}, m g l\right)=(1.0,2.2,2.7,0.46)$, respectively. Therefore, the initial estimation values are deliberately chosen to be considerably different than the actual values. The process and measurement noise covariance matrices used in state and parameter estimators are

$$
\begin{aligned}
& R_{\text {state }}= \operatorname{diag}([1 e-10,1 e-10,1 e-10,0]) ; \\
& Q_{\text {state }}= \operatorname{diag}([1 e-8,1 e-8,1 e-8,1 e-8, \\
&1 e-7,1 e-7,1 e-7]) ; \\
& Q_{\text {parameter }}= \operatorname{diag}([1 e-5,1 e-5,1 e-5,1 e-5]) ; \\
& R_{\text {parameter }}= \operatorname{diag}([1 e-8,1 e-8,1 e-8,1 e-8, \\
&1 e-7,1 e-7,1 e-7]) .
\end{aligned}
$$

\section{A. Results and Analysis}

The UKF estimation results for the system parameters and the corresponding estimated states are shown in Figs. 2-4. The true values are the solid lines and the estimated values are the dotted, dashed, and dash-dotted lines that are used to differentiate various states. The figures show that, despite such a large initial errors, the system states and parameters converge to their real values. As expected for the aboveexplained dual algorithm, parameter convergence is achieved at a much slower rate $(1500 \mathrm{~s})$ than the state convergence (200 s).

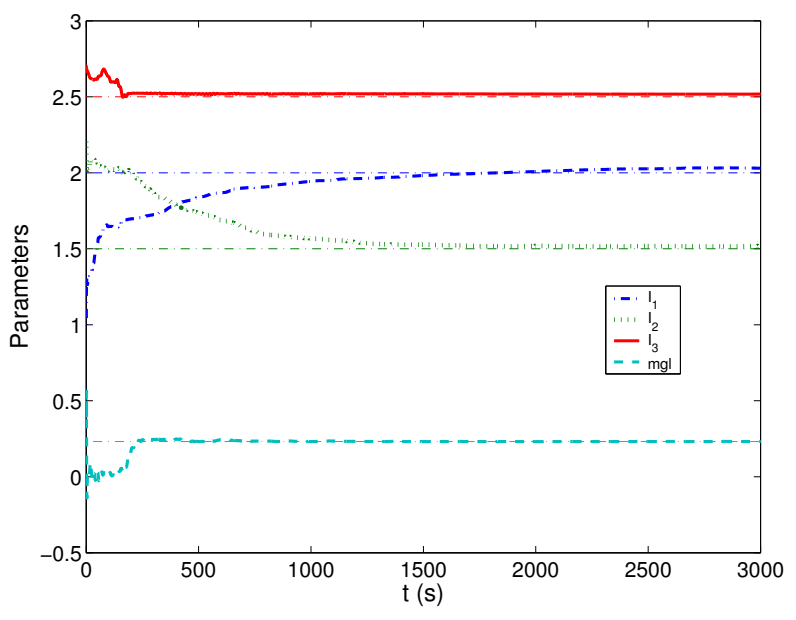

Fig. 2. Parameter estimation.

To further examine the states convergence, the norm of the estimated quaternions is shown in Fig. 5. The figure illustrates that the unit quaternion constraint is only enforced after the state convergence is achieved. As a last measure of ensuring convergence, we calculate and compare the values of the Earth magnetic field (eq (9)) using the true and estimated state values. If convergence is achieved, the results should be in good agreement. Fig. 6 shows the results where the estimated values are overlayed on top of the true values in black solid line. The excellent match between the two is another indication that the estimation scheme has, indeed, converged.

\section{GROUnd TEST EXPERIMENTS}

In this section, the actual magnetometer measurements collected throughout the airbearing maneuver is used for the dual state-parameter estimation. The intension is to study the performance of the method in real test experiments. 


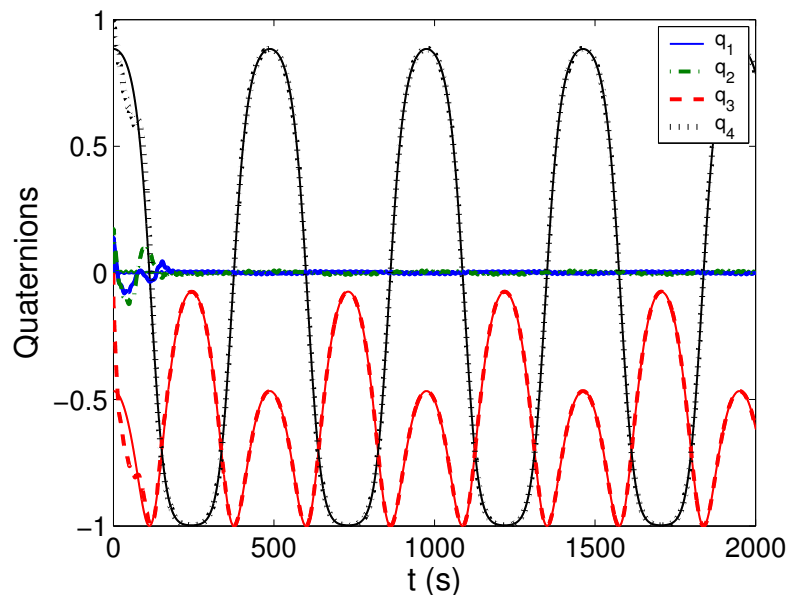

Fig. 3. Quaternion estimation. Estimated values are overlayed on top of the true values in solid line.

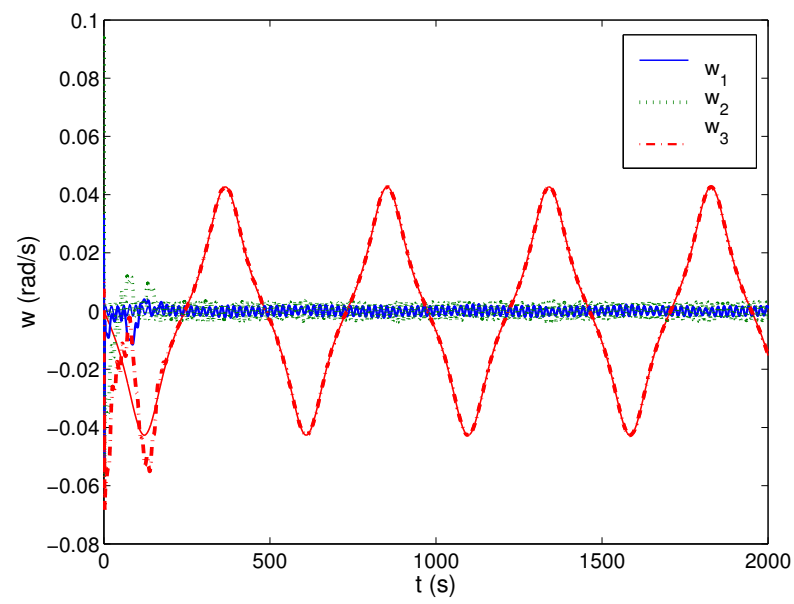

Fig. 4. Angular velocity estimation. Estimated values are overlayed on top of the true values in solid line.

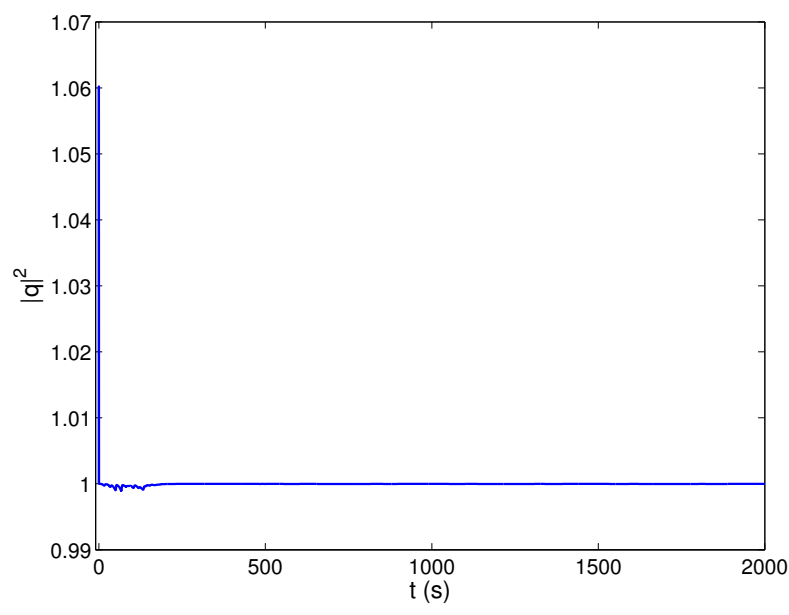

Fig. 5. The norm of the estimated quaternions.

\section{A. Experimental Test Rig}

The air-bearing test rig (Fig. 7) offers free motion about three perpendicular axes replicating the NPSAT1 attitude

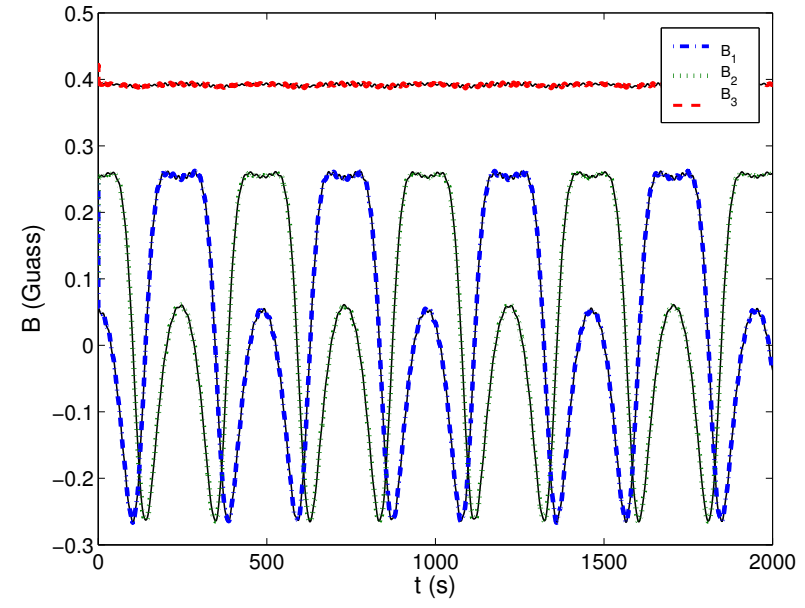

Fig. 6. The Earth magnetic field. Estimated values are overlayed on top of the true values in black solid line.

motion on Earth. It is equipped with on-board power supply, three MT-30-2-CGS Microcosm magnetic torque rods, and a Honeywell Smart Digital Magnetometer HMR2300 aligned with the system's principal axes. The magnetometer measures the Earth's instantaneous magnetic field in each $\mathrm{x}, \mathrm{y}$, and $z$ direction. The onboard single-board computer (SBC) is a repackaged PC running a $400 \mathrm{MHz}$ Pentium. It interfaces with the magnetometer and the torque control board via serial commands to a simple onboard microcontroller. The SBC's Input/Output (I/O) is orchestrated with a command generation laptop and accepts ASCII-encoded serial-based (RS232) commands. The serial communication is through a Linksys wireless Ethernet bridge and using Matlab Instrument Control Toolbox. The overall platform is passively balanced by dummy weights installed symmetric to magnetic torque rods, magnetometer, wireless bridge, etc.

In order to validate the UKF-based estimated states, the air-bearing is furnished with an optical measurement system that can directly measure the attitude. It consists of an onboard laser diode, an indicator screen, a CCD camera and a PC loaded with analysis software that converts the camera readings into Euler angles.

\section{B. Results and Analysis}

Similar to simulation analysis, a constant control of

$$
u_{1}=u_{2}=-u_{3}=-33 A / m^{2}
$$

is applied to airbearing table. The maneuver starts when the system is oriented near $\left(q_{1}, q_{2}, q_{3}, q_{4}\right)=(0,0,-0.47,0.88)$ with $\left(\omega_{x}, \omega_{y}, \omega_{z}\right)=(0,0,0) \mathrm{rad} / \mathrm{s}$. The only sensor measurement available to the UKF is the magnetic field measured by the magnetometer. Estimation routine starts from $\left(q_{1}, q_{2}, q_{3}, q_{4}\right)=(0,0,0,1)$, and $\left(\omega_{x}, \omega_{y}, \omega_{z}\right)=$ $(-0.02,0.02,0.01) \mathrm{rad} / \mathrm{s}$ which implies large initial errors in orientation and angular rates. In the absence of a means to verify the converged values of the system parameters, various values are used as the parameters initial estimates spanning over [1.0-2.5] for $I_{1}$ and $I_{2}$, [2.1-2.7] for $I_{3}$ and [0.01-0.09] 


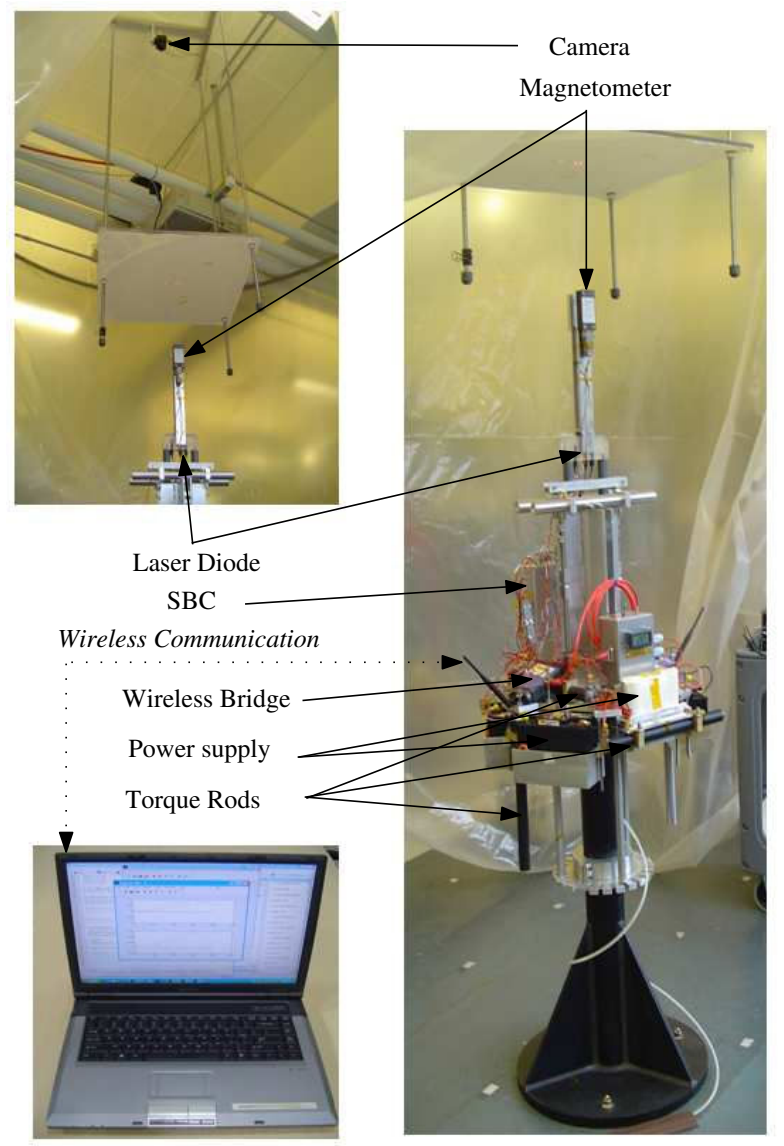

Fig. 7. Air-bearing test rig.

for $m g l$. The process and measurement covariance matrices used in the dual state-parameter estimation are:

$$
\begin{aligned}
R_{\text {state }}= & \operatorname{diag}([1 e-8,1 e-8,1 e-8,0]) ; \\
Q_{\text {state }}= & \operatorname{diag}([1 e-7,1 e-7,1 e-7,1 e-7, \\
& 1 e-7,1 e-7,1 e-7]) ; \\
Q_{\text {parameter }}= & \operatorname{diag}([1 e-2,1 e-2,1 e-4,1 e-6]) ; \\
R_{\text {parameter }}= & \operatorname{diag}([1 e-5,1 e-5,1 e-5,1 e-5, \\
& 1 e-3,1 e-3,1 e-4]) .
\end{aligned}
$$

UKF estimation results for the system parameters is depicted in Fig. 8. It clearly indicates that, regardless of the initial conditions, the estimated values for the parameters converge to $\left(I_{1}, I_{2}, I_{3}, m g l\right)=(1.4,1.5,2.5,0.05)$, respectively.

The convergence of the estimation scheme is verified in a variety of different and/or completely independent ways. The first verification method is to compare the UKF estimated quaternions with the attitude measurements obtained from the onboard optical measurement system. Although quaternion or angular rates will not be directly measured in orbit, the ground test-bed is equipped with a CCD camera capable of recording the Euler angles $(\theta, \phi, \psi)$ throughout the motion. The Euler angles are then converted to quaternions

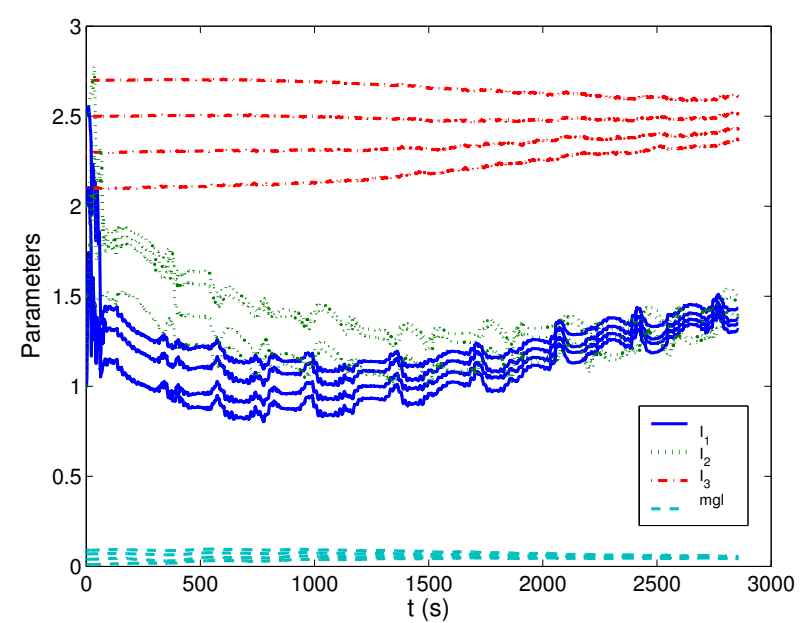

Fig. 8. Estimated parameters.

according to the following equation:

$$
\left[\begin{array}{l}
q_{1} \\
q_{2} \\
q_{3} \\
q_{4}
\end{array}\right]=\left[\begin{array}{c}
\sin (\theta / 2) \cos ((\phi-\psi) / 2) \\
\sin (\theta / 2) \sin ((\phi-\psi) / 2) \\
\cos (\theta / 2) \sin ((\phi+\psi) / 2) \\
\cos (\theta / 2) \cos ((\phi+\psi) / 2)
\end{array}\right]
$$

Fig. 9 depicts the estimated vs directly measured quaternions. It shows that, when convergence is achieved, the UKF-estimated and camera-measured quaternions coincides within the camera's precision of $2.5^{\circ}$. As a next measure of

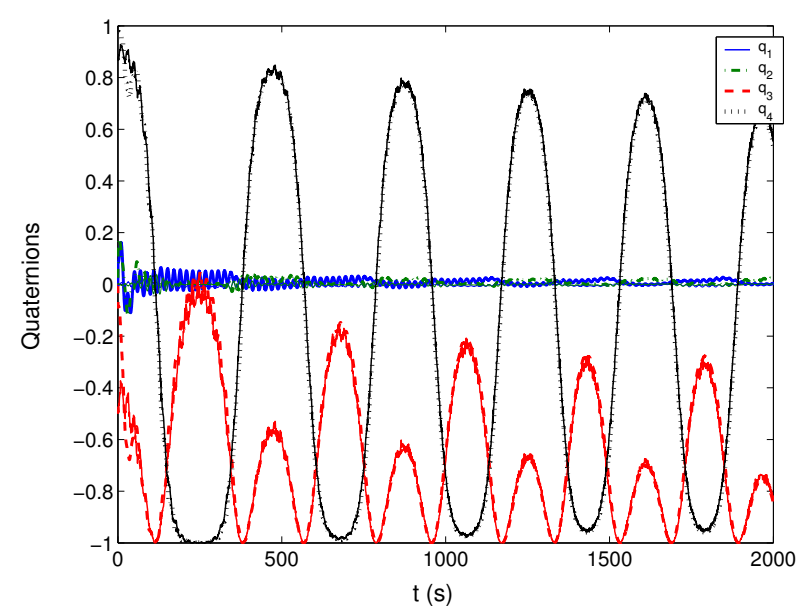

Fig. 9. Quaternion estimation. Estimated values are overlayed on top of the directly measured values in solid lines.

convergence, the Earth magnetic field is calculated using the UKF estimated states [see (9)] and compared to the actual magnetometer readings. Fig. 10 demonstrates the excellent match between the two estimation-based values and the actual measurements, thus, verifying the convergence of the UKF algorithm. The last convergence test is to check the unity of the estimated quaternion norm. This, is depicted in Fig. 11. The figure confirms that, when convergence is achieved, the unity of the quaternion is preserved throughout the estimation. 


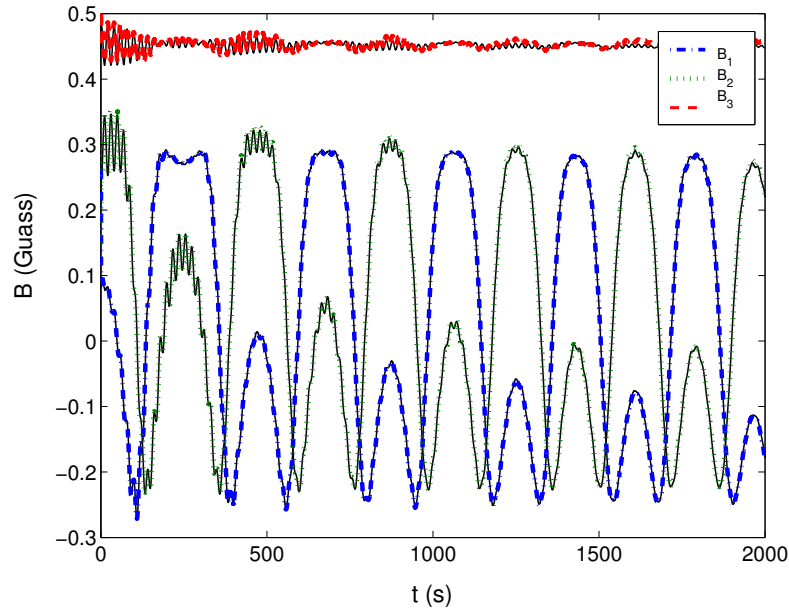

Fig. 10. The Earth magnetic field. Estimated values are overlayed on top of the actual magnetometer readings in black solid lines.

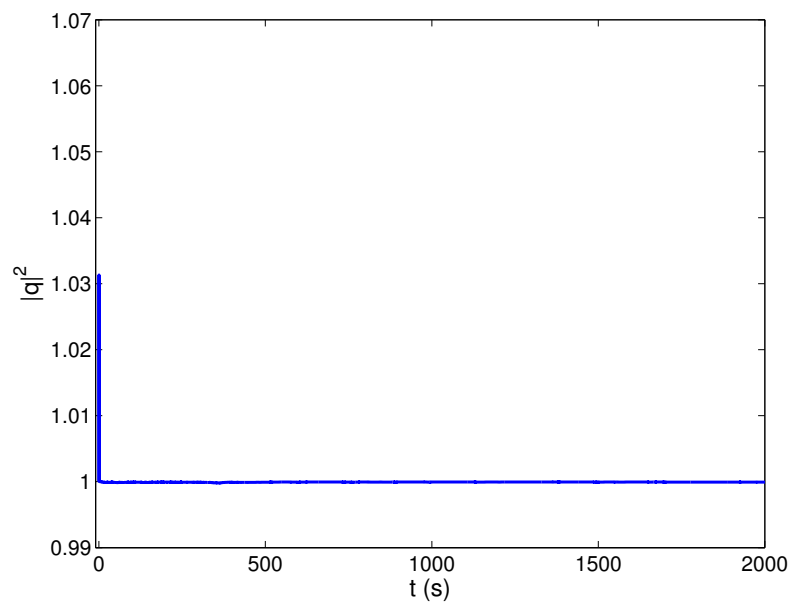

Fig. 11. The norm of the estimated quaternion.

Finally, it worths reminding the readers that the above UKF dual estimation algorithm also provides estimated values of other unmeasurable states such as angular rates, as shown in Fig. 12.

\section{CONCLUSIONS}

Unscented Kalman Filter was successfully implemented for parameter estimation of the NPSAT1 in-house fabricated ground test assembly. Despite the inherently nonlinear dynamics of the system, both simulation and experimental results showed that the UKF dual state-parameter estimation scheme is capable of estimating the true values of states and parameters. The convergence of the method was confirmed in various ways, including an independent attitude measurement using an onboard optical measurement system.

\section{ACKNOWLEDGMENTS}

The authors are grateful to Jim Horning, Dan Sakoda and Ron Phelps for their assistance in the experimental setup and interfacing. This research was supported in part by AFOSR

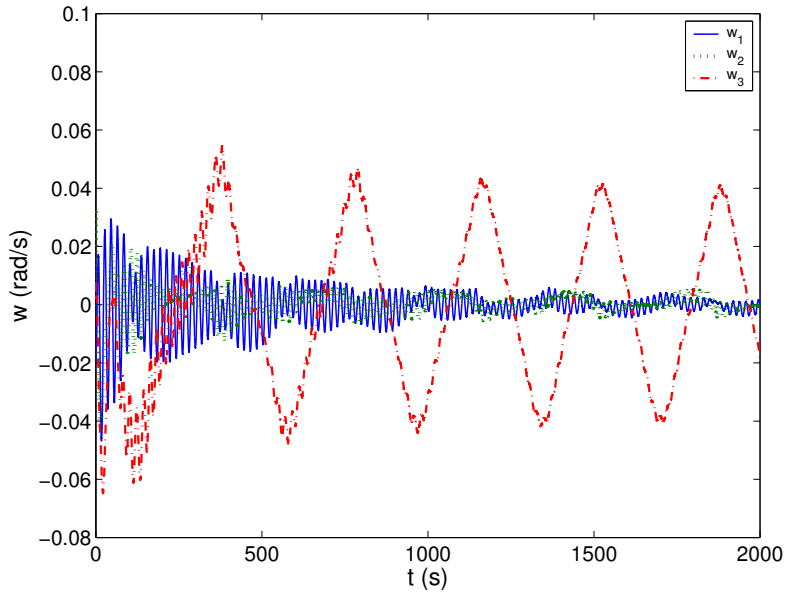

Fig. 12. Estimated angular velocities.

under Grant No F1ATA0-60-6-2G002 and the Secretary of the Air Force.

\section{REFERENCES}

[1] E. J. Lefferts, F. L. Markley and M. D. Shuster, Kalman Filtering for Spacecraft Attitude Estimation, Journal of Guidance, Control, and Dynamics, Vol. 5, No. 5, 1982, pp. 417-429.

[2] J. K. Deutschmann, I. Y. Bar-Itzhack, Evaluation of Attitude and Orbit Estimation Using Actual Earth Magnetic Field Data, Journal of Guidance, Control, and Dynamics, Vol. 24, No 3, 2001, pp. 616-623.

[3] M. L. Psiaki and Y. Oshman, Spacecraft Attitude Rate Estimation from Geomagnetic Field Measurements, Journal of Guidance, Control, and Dynamics, Vol. 26, No 2, 2003, pp. 244-252.

[4] S. J. Julier and J. K. Uhlmann, Unscented filtering and nonlinear estimation, Proceedings of the IEEE, Vol. 92, No. 3, 2004, pp. 401422.

[5] S. J. Julier, J. K. Uhlmann and H. F. Durrant-Whyte, A new method for the nonlinear transformation of means and covariances in filters and estimator, IEEE Trans. Automatic. Control., Vol. 45, No. 3, pp. 477-482, 2000.

[6] J. L. Crassidis and F. L. Markley, Unscented filtering for spacecraft attitude estimation, AIAA Journal on Guidance, Control, and Dynamics, Vol. 26, No. 4, 2003, pp. 536-542.

[7] F. Daum, Nonlinear filters: beyond the Kalman Filter, IEEE A\&E Systems Magazine, Vol. 20, No. 8, 2005, pp. 57-69.

[8] M. C. VanDyke, J. L. Schwartz, C. D. Hall, Unscented Kalman filtering for spacecraft attitude state and parameter estimation, AAS-04-115, 2004.

[9] E. Kraft, A Quaternion-based Unscented Kalman Filter for Orientation Tracking, Proceedings of the 6th International Conference on Information Fusion, Cairns, Australia, 2003, pp. 47-54.

[10] P. Sekhavat, Q. Gong and I. M. Ross, Unscented Kalman filtering: NPSAT1 ground test results, AIAA Guidance, Navigation, and Control Conference, Keystone, Colorado, Aug., 2006.

[11] R. F. Stengel, Optimal Control and Estimation. Dover Publications Inc., Sep. 1994.

[12] A. Sitz, U. Schwartz, and J. Kurths, The Unscented Kalman Filter, A Powerful Tool for data Analysis, International Journal of Bifurcation and Chaos, Vol. 14, No 6, 2004, pp. 2093-2105.

[13] E. A. Wan, R. van der Merwe, and A. T. Nelson, Dual Estimation and the Unscented Transformation, Advances in Neural Information Processing Systems 12, MIT Press, 2000, pp. 666672.

[14] L. W. Nelson and E. Stear, The Simultaneous On-Line Estimation for Parameters and States in Linear Systems, IEEE Transactions on Automatic Control, Vol. AC-21, No. 2, pp. 9498, 1976.

[15] A. Fleming, Real-time optimal slew maneuver design and control, Astronautical Engineer's Thesis, US Naval Postgraduate School, December 2004.

[16] J. R. Wertz, Spacecraft Attitude determination and control, Kluwer Academic Publishers, 1978. 